\title{
Angiographic variation of the internal carotid artery and its branches in horses
}

\begin{abstract}
To record the angiographic anatomy of the equine internal carotid artery (ICA) using angiography techniques. In vitro descriptive study. Equine cadaver specimens $(n=50)$. Head and neck specimens from horses of mixed breed, age, sex, and use without a history of guttural pouch disease had carotid and cerebral angiography using conventional $(n=7)$ and rotational angiography (43). Angiographic findings were verified by arterial latex casts. Variation in ICA anatomy was categorized into 4 groups: (1) the internal carotid and occipital arteries arising as a common trunk; (2) an aberrant branch of the extra-cranial ICA connected to the basilar artery; (3) an aberrant branch of the ICA ramifying into the surrounding tissue and not connected to any other vessels; and (4) an aberrant branch of the ICA giving rise to several smaller satellite branches, including connections to the caudal branch of the ipsilateral occipital artery. Rotational angiography is useful for identification of anatomic variation in the ICA that could be important in achieving vascular occlusion in the treatment of guttural pouch mycosis
\end{abstract}

